\title{
Transient vibrational circular dichroism spectrometer: technical development
}

\author{
M. Bonmarin and J. Helbing
}

Physikalich-Chemisches Institut, 8057, Zurich, Switzerland

\begin{abstract}
We recently reported the first measurements of transient VCD in the C-H-stretch region following visible excitation of cobalt(-)-spartein complex $(\mathrm{Co}(\mathrm{sp}) \mathrm{Cl} 2)$ with picosecond time resolution [1]. This poster presents the detailed description of the setup based on the synchronization of a femtosecond laser system with a photo elastic modulator [2]. A very precise control of the probe pulse polarization is a requirement to avoid linear dichroism artefacts. This is particularly important in crossed polarizer "quasi-null" technique which can be used to significantly enhance chiral signals [3].
\end{abstract}

\section{References}

1. M. Bonmarin and J. Helbing, Optics Letters, 33, 2086 (2008)

2. M. Bonmarin and J. Helbing, Chiriality (to be published)

3. J. Helbing and M. Bonmarin, J. of Chem. Phys., 131, 174507 (2009) 